\title{
Archéopages
}

Archéopages

Archéologie et société

40 | 04-07/2014

Villages

\section{Construire au Moyen Âge en région Centre}

Économie de la pierre et techniques de construction du XII ${ }^{\mathrm{e}} \mathrm{au} \mathrm{XVI} \mathrm{I}^{\mathrm{e}}$ siècle. L'apport des chantiers d'archéologie préventive

\section{Simon Bryant}

\section{CpenEdition}

\section{Journals}

Édition électronique

URL : https://journals.openedition.org/archeopages/667

DOI : 10.4000/archeopages.667

ISSN : 2269-9872

\section{Éditeur}

INRAP - Institut national de recherches archéologiques préventives

\section{Édition imprimée}

Date de publication : 1 juin 2015

Pagination : 176-177

ISSN : 1622-8545

\section{Référence électronique}

Simon Bryant, «Construire au Moyen Âge en région Centre », Archéopages [En ligne], 40 | 04-07/2014, mis en ligne le 01 juillet 2016, consulté le 23 janvier 2022. URL : http://journals.openedition.org/ archeopages/667 ; DOI : https://doi.org/10.4000/archeopages.667 


\section{Simon Bryant}

21 MARS 2014

Université Paris I,

Panthéon-Sorbonne

Directrice de thèse:

Joëlle Burnouf

Professeure émérite d'archéologie

médiévale, Université Paris I

Membres du Jury:

Florence Journot,

Maître de conférences,

Université Paris I

Bruno Phalip,

Professeur d'Histoire de l'art

et d'archéologie médiévale,

Université de Clermont-Ferrand

Patrick Ponsot,

Architecte en chef

des Monuments historiques

Christian Sapin,

Directeur de recherches, CNRS,

UMR 6298 ARTeHIS,

Université de Dijon

Jean Terrier,

Professeur, Unité d'Archéologie

classique, Université de Genève
Inrap, UMR 7041 «Archéologie et sciences de l'Antiquité»

\section{Construire au Moyen Âge en région Centre}

\author{
Économie de la pierre et techniques \\ de construction du $x \|^{e}$ au $x v l^{e}$ siècle. L'apport \\ des chantiers d'archéologie préventive
}

Ce mémoire constitue une mise en perspective de presque vingt ans d'interventions archéologiques réalisées dans le cadre des chantiers de restauration et d'aménagement du «patrimoine architectural et archéologique». On estime avant tout qu'il s'agit de vestiges avant d'être considérés ou non comme du «patrimoine». La première partie est composée d'une réflexion sur le cadre réglementaire et institutionnel qui détermine quand et comment l'archéologie peut intervenir dans un contexte où les processus gouvernant la recherche archéologique se juxtaposent avec ceux de la protection, de la restauration et de l'aménagement de ce patrimoine. Néanmoins, le cadre juridique offre une diversité de réponses possibles à des situations où ces vestiges peuvent être concernés par les projets d'aménagement. Si l'intégration de l'archéologie dans ces derniers n'est pas encore à la hauteur du potentiel offert par les chantiers de restauration, la preuve tangible de la réussite des collaborations et des dialogues positifs a été présentée sous la forme d'un bilan régional et thématique de plus de 183 chantiers réalisés dans la région depuis une vingtaine d'années.

Ces interventions devaient répondre en premier lieu aux attentes des acteurs chargés de la gestion du patrimoine. Ce faisant, elles ont produit une quantité importante d'observations secondaires par rapport aux objectifs principaux. Souvent inexploitées, elles constituent une source riche de données scientifiques intéressantes pour la recherche archéologique. La deuxième partie du travail consiste en une évaluation critique de la méthodologie mise en œuvre lors des interventions, dont une présentation de la documentation écrite et iconographique exploitée pour chaque site. La diversité des cadres juridiques ont conduit à des approches et à des méthodes très différentes, conditionnant en partie la qualité et la quantité des données obtenues. La recomposition des rapports de fouille pour notre corpus fut une confrontation directe avec les problèmes de la pérennité et de la transmission des données archéologiques et de la qualité de l'archive principale. Cette partie du travail a permis d'établir le bilan régional et de présenter le corpus des sites qui servent de base pour la troisième partie du mémoire: 41 édifices de différents types de la région Centre ayant fait l'objet d'interventions archéologiques sous la responsabilité de l'auteur. Avec une fourchette chronologique comprise entre le milieu du XI ${ }^{e}$ siècle et le début du XVII siècle, la composition de ce corpus est le reflet de la politique de protection et d'aménagement du patrimoine. Certaines catégories d'édifice sont donc sous-représentées, notamment l'habitat domestique rural et urbain. Ce corpus fut complété par 39 édifices, essentiellement des églises paroissiales, dans le sud du Berry. Ces édifices servent de points de comparaison.

La composition d'apparence hétéroclite du corpus peut être vue comme une forme d'échantillonnage qui permet de s'affranchir des typologies habituelles ou des fourchettes chronologiques fermées. Cette diversité obligeait l'adoption d'une approche comparative et diachronique, où les inévitables lacunes propres à chaque site étaient atténuées par la multiplication du nombre de sites. L'exploitation des données des fouilles a permis de restituer la chaîne opératoire du chantier de construction de la carrière jusqu'à l'achèvement de l'édifice et son évolution jusqu'à l'objet patrimoine d'aujourd'hui. Les compétences des bâtisseurs ont été appréhendées par plusieurs biais dont l'exploitation du potentiel offert par l'environnement géologique et les soins apportés à la préparation et à la mise en œuvre de la pierre. Les relations entre le petit appareil et la pierre de taille ont été considérées sous la lumière des considérations économiques, techniques et esthétiques.

L'analyse des techniques de fondation et des rapports entre les composants du gros œuvre (maçonneries, ouvertures, systèmes de couvrement) permet d'évaluer le savoir-faire des bâtisseurs. La pérennité de certaines formes et techniques et la récurrence de certains désordres pendant toute la période chronologique ont été constatées et posent la question de la définition de ce qui peut être considéré comme une architecture «savante» par rapport à celle dite «traditionnelle», sous-entendu peu avancée ou archaïque. Ces termes deviennent plutôt relatifs, voire inopérants. Face à une évolution des compétences qui n'était ni universelle ni linéaire, les relations entre bâtisseur, commanditaire et la technicité d'un édifice se sont montrées plus complexes. La taille d'un édifice ou le statut élevé de son commanditaire ne garantissent nullement une conception technique sans faute ni une exécution soignée. Si la place de la pierre dans la construction a été prépondérante dans notre corpus, il a été possible de souligner l'importance du bois à toutes les étapes de la construction et à toutes les périodes. Parfois préservé in situ, où il est une source potentielle de données chronologiques et d'indicateurs environnementaux, la restitution des structures disparues passe essentiellement par l'analyse des négatifs que laisse le bois dans les maçonneries. Pour certains sites, la maçonnerie visible aujourd'hui n'est qu'une armature pour des ouvrages en bois. La lecture des traces du chantier au sol et dans les élévations permet d'aborder les processus de la construction. Pour le travail en hauteur, il a été possible d'esquisser un 
panorama des aménagements provisoires du chantier où, encore une fois, le bois joue un rôle prépondérant. Couplées avec l'analyse des parements, ces structures provisoires permettent d'identifier des séquences de montage des maçonneries, des ruptures dans le travail ou des changements de projet.

À travers la synthèse de ces données, il a été possible de toucher aux aspects sociaux du chantier dont l'organisation du travail et des éventuelles expressions de l'individualité des ouvriers ou d'une équipe. Les techniques de montage et l'interprétation des marques lapidaires posent la question de la mobilité des artisans et la transmission du savoir-faire, mais cet aspect du chantier a sans doute été insuffisamment développé. La dimension humaine reste moins facilement abordable que les aspects techniques. Sans doute faudrait-il d'autres données pour mieux cerner les bâtisseurs restés encore en filigrane. La question du cadre de vie a été abordée par le biais des observations sur les éléments de finition comme les revêtements au sol, les enduits, les éléments de confort et d'hygiène. Les aléas de la conservation ont toutefois tendance à nous laisser avec l'impression d'une coquille vide alors que le bâti ancien sous toutes ses formes était garni par le second œuvre des différents corps de métier et par un mobilier parfois riche. Cet aspect de notre travail, et l'appréciation du « comment vivre» méritent également d'être approfondis.

En appréhendant le chantier dans sa matérialité, l'approche archéologique permet d'écrire une histoire de la construction parfois bien différente quoique complémentaire à celle connue de l'analyse stylistique ou de l'étude des textes. L'exploitation de ces données démontre que l'archéologie représente une véritable plus-value non seulement pour les besoins de la gestion du patrimoine architectural, mais aussi pour la recherche scientifique. Elle a également démontré tantôt par sa présence tantôt par son absence, l'importance du concours des autres disciplines et d'un regard pluriel pour passer du stade de la mise en phase et de la description, aussi complètes soient-elles, à celui d'une interprétation approfondie: historiens de l'art, de l'architecture et de la religion, les spécialistes des matériaux, ethnologues et hommes de métier. Le chantier de restauration ou d'aménagement reste toujours un lieu privilégié où sont réunis les moyens matériels et humains et la possibilité d'accéder à notre objet d'étude. Les méthodologies mises en œuvre dans ce contexte sont certes puisées dans le répertoire commun à tous les archéologues. Cependant, les enjeux qui lui sont propres nécessitent encore de trouver des réponses institutionnelles pour améliorer la prise en compte de notre discipline.
Marion Saurel

Inrap, UMR 8546

8 AVRIL 2014

Université Paris I, PanthéonSorbonne, École doctorale d'archéologie (ED 112)

Directeur de thèse :

Jean-Paul Demoule,

Professeur, Université Paris I

Membres du Jury:

Rapporteurs:

Philippe Barral,

Professeur, Université

de Franche-Comté

Marie-Yvane Daire,

Directrice de recherche, CNRS,

Experts :

Bernard Lambot,

Archéologue bénévole

Jeannot Metzler,

Conservateur, MNHA Luxembourg

SAUREL M., à paraître: La céramique à Acy-Romance et dans le pays rème (vers 300 à 30 avant J.C.) descriptions et hypothèses fonctionnelles, in Mémoires de la Société archéologique champenoise (Monographie du site protohistorique d'AcyRomance (Ardennes); VI).
«Archéologie et philologie d'Orient et d'Occident »

\section{La céramique à Acy-Romance et dans le pays rème (vers 300 à $\mathbf{3 0}$ av. J.-C.).}

\section{Descriptions et hypothèses fonctionnelles.}

L'apport de la céramique à la connaissance des pratiques sociales de lâge du Fer passe par une approche concrète de l'utilisation des récipients et ustensiles. Si l'expérimentation et les analyses chimiques, notamment de matières biologiques, en plein développement, sont appelées à jouer un rôle décisif, elles sont indissociables d'une étude fonctionnelle systématique des restes céramiques intégrant l'ensemble des données - la morphologie, la technique, la capacité, les traces, mais aussi les contextes et l'environnement au sens large.

Les principaux corpus traités sont issus d'une part, de la fouille programmée d'Acy-Romance dirigée par Bernard Lambot et d'autre part, de fouilles préventives de l'Inrap sur le parcours du TGV Est. Les sites appartiennent au nord de la plaine crayeuse de Champagne, entre le cours de l'Aisne que surplombe le village d'Acy-Romance occupé aux IIe-Ier siècle avant notre ère et la vallée de la Vesle où se trouvent les habitats de Champfleury et de Villers-aux-Nœuds, datés des IVe-IIe siècles. Ils s'inscrivent au cœur de ce qui fut à la fin de l'âge du Fer le domaine des Rèmes quelles que soient les fluctuations envisagées pour cette entité territoriale.

Le premier volet, consacré à la périodisation de la céramique d'Acy-Romance dans son cadre régional, permet de cerner l'évolution de la production céramique et de replacer les phénomènes dans une perspective historique. Les logiques internes à cette évolution recoupent les phases de l'histoire du village du premier temps d'inventivité foisonnante autour du milieu du Ile siècle avant notre ère en accord avec une agglomération dynamique, à une période de basculement puis à une standardisation des procédés et des formes concomitantes au développement des oppida et à la perte d'importance du site à l'échelle du pays rème. Cette réflexion repose sur deux classements morphologique et technique - élaborés de manière à s'accorder avec l'approche fonctionnelle et apporte une maîtrise relative de la donnée « temps » qui constitue un préliminaire indispensable.

La première étape est celle de la mise en place des outils spécifiques à l'analyse fonctionnelle; tout d'abord, le classement des récipients et ustensiles du corpus en «familles» (d'après la technique, la morphologie et la capacité) et, en second lieu, le classement raisonné des traces liées à l'emploi. Chaque famille et chaque catégorie de traces font l'objet d'un bilan documentaire afin de déterminer un premier éventail des possibles. Deuxièmement, le croisement des données correspond à une analyse systématique de la représentation des traces pour chaque famille, et au recoupement des 